\title{
Beam Test Results of the ISS-CREAM Calorimeter
}

\author{
H.G Zhang, ${ }^{a, *}$ D. Angelaszek, ${ }^{a, b}$ M. Copley, ${ }^{a}$ J.H. Han,${ }^{a}$ H.G. Huh, ${ }^{a}$ Y.S. Hwang,,$e$ \\ H.J. Hyun, ${ }^{c, h}$ H.B. Jeon, ${ }^{c}$ K.C. Kim, ${ }^{a}$ M.H. Kim,${ }^{a}$ H.J. Kim, ${ }^{c}$ K. Kwashnak, ${ }^{a}$ M.H. \\ Lee, ${ }^{a, d}$ J.P. Lundquist, ${ }^{a}$ L. Lutz, ${ }^{a}$ A. Malinin, ${ }^{a}$ H. Park, ${ }^{c}$ J.M. Park, ${ }^{c, f}$ N. \\ Picot-Clemente, ${ }^{a}$ E.S. Seo,${ }^{a, b}$ J. Smith, ${ }^{a}$ J. Wu, ${ }^{a}$ Z.Y. Yin ${ }^{a}$ and Y.S. Yoon ${ }^{a, g}$ \\ ${ }^{a}$ Institute for Physical Science and Technology, University of Maryland, College Park, MD 20742, USA \\ ${ }^{b}$ Department of Physics, University of Maryland, College Park, MD 20742, USA \\ ${ }^{c}$ Department of Physics, Kyungpook National University, Daegu 41566, Korea \\ ${ }^{d}$ Center for Underground Physics, Institute for Basic Science (IBS), Daejeon 34126, Korea \\ ${ }^{e}$ Korea Atomic Energy Research Institute, Gyeongju 38180, Korea \\ ${ }^{f}$ Advanced Radiation Technology Institute, Korea Atomic Energy Research Institute, Jeongeup 56212, \\ Korea \\ ${ }^{g}$ Korea Research Institute of Standards and Science, Daejeon 34113, Korea \\ ${ }^{h}$ Pohang Accelerator Laboratory, Pohang 37673, Korea \\ E-mail: zhanghg.umd@protonmail.com
}

The Cosmic Ray Energetics And Mass experiment for the International Space Station (ISSCREAM) was installed on the ISS to measure high-energy cosmic-ray elemental spectra for the charge range $\mathrm{Z}=1$ to 26 . The ISS-CREAM instrument includes a tungsten scintillatingfiber calorimeter preceded by carbon targets for energy measurements. The carbon targets induces hadronic interactions, and showers of secondary particles develop in the calorimeter. The calorimeter was calibrated with electron beams at CERN. This beam test included position, energy, and angle scans of electron and pion beams together with a high-voltage scan for calibration and characterization. Additionally, an attenuation effect in the scintillating fibers was studied. In this paper, beam test results, including corrections for the attenuation effect, are presented.

$37^{\text {th }}$ International Cosmic Ray Conference (ICRC 2021)

July 12 th - 23rd, 2021

Online - Berlin, Germany

\footnotetext{
${ }^{*}$ Presenter
} 


\section{The structure of the calorimeter}

The ISS-CREAM calorimeter consisting of a carbon target, twenty tungsten plates and twenty scintillating-fiber ribbons layers (see [1-3]), is shown in Fig. 1 with a cross-sectional view. The carbon target, of $19.8 \mathrm{~cm}$ height, initiates hadronic showers upstream with two densified graphite blocks. The tungsten plates, each of $3.5 \mathrm{~mm}$ thick $50 \mathrm{~cm} \times 50 \mathrm{~cm}$, are below the target. A layer with fifty fiber ribbons is placed between each consecutive tungsten layer covering the same area as the tungsten plate. The fiber ribbon is made of nineteen $0.5 \mathrm{~mm}$ diameter scintillating fibers with a width of $1 \mathrm{~cm}$, while every other ribbon is mated with light guides on one side of the calorimeter and the other half are mated to the opposite side. The scintillation light, which is generated in the fibers by charged particles from the shower, is transmitted to a hybrid photodiode via a light mixer and a bundle of transparent fibers [4].

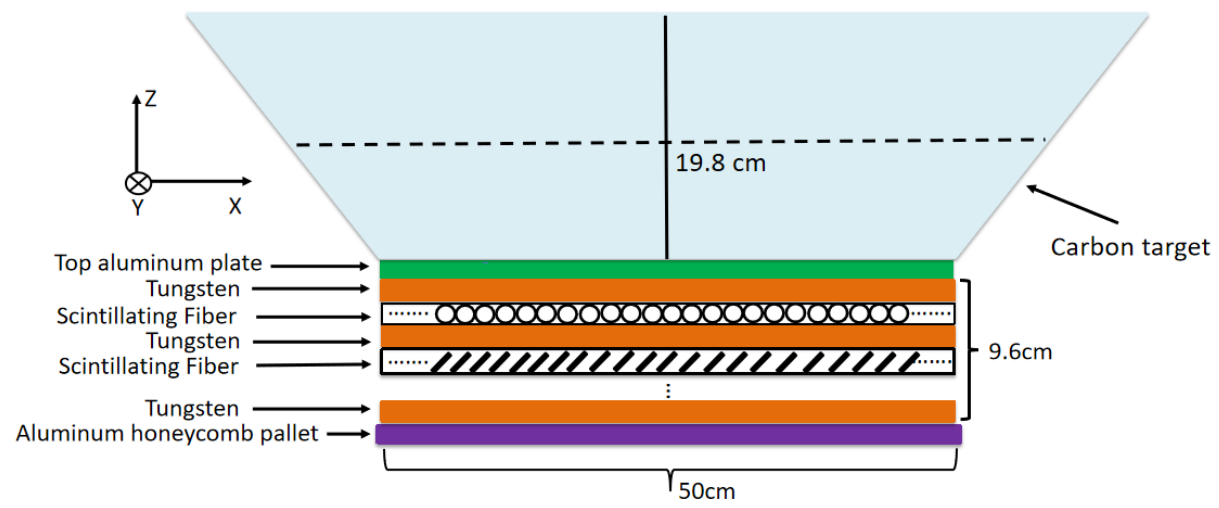

Figure 1: A cross-sectional view of the calorimeter and the carbon target in the beam test configuration.

\section{The beam test scan}

Four detector configurations were implemented to measure the ribbon response throughout the calorimeter. In the "No-Lead-Front" configuration, the electron beam was injected into the front of the graphite target from the $+Z$-direction, while in the "No-Lead-Rear" configuration, the electron beam was incident on the bottom of the detector from the -Z-direction by rotating the detector. In order to maximum the shower in the first few, and the last few layers, $2.5 \mathrm{~cm}$-thick lead bricks were placed in front of the calorimeter, in the "Lead-Front" and "Lead-Rear" configurations (see Fig.2).

The energy deposit to each ribbon's beam response, is estimated in the Monte Carlo simulation. The calibration factor on each ribbon is generated with comparing the ADC from beam test measurement and $\mathrm{MeV}$ from the Monte Carlo simulation. Additional corrections to the calibration factor were made, including attenuation corrections and gain corrections.

The ribbon is calibrated with a $150 \mathrm{GeV}$ electron beam, which has a Gaussian distribution profile with a standard deviation of $\sim 4.6 \mathrm{~mm}$ [5]. Ribbons in the 22 nd position from the $-\mathrm{X}$ side of odd layers in the calorimeter were exposed to beams as shown in Fig. 3. The calorimeter was moved by $1 \mathrm{~cm}$ along the X-direction to expose the next ribbon center to the beam. Measurements were repeated for all fifty ribbons in the odd layers, and similarly, the measurement was moved by $1 \mathrm{~cm}$ along the Y-direction to expose the even layer ribbon centers to the beams. 

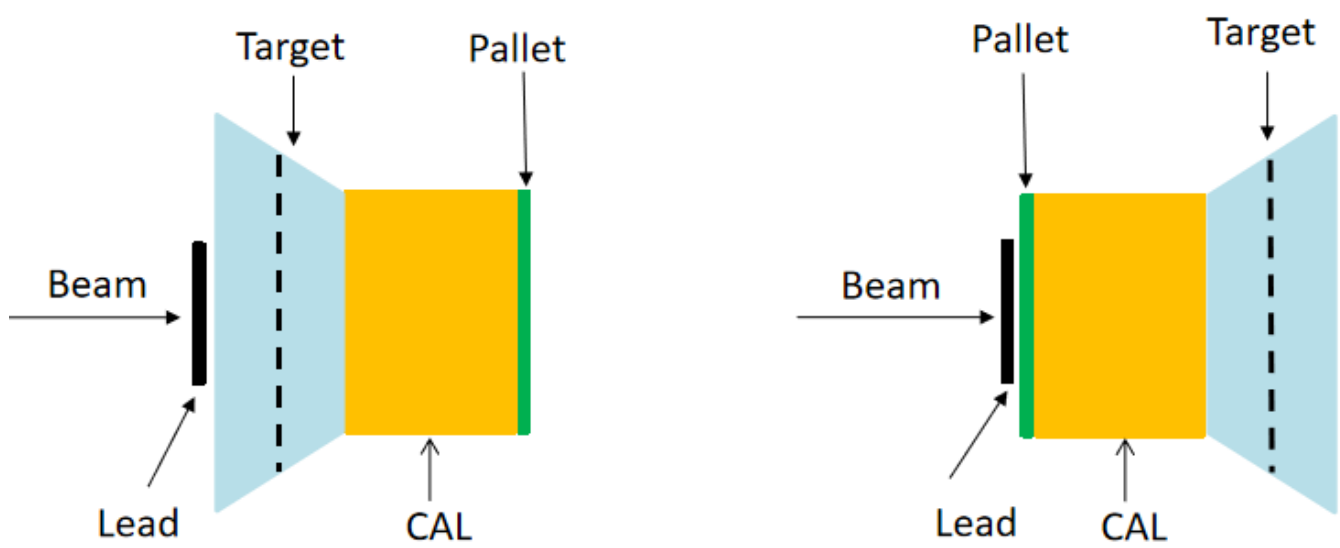

Figure 2: The configurations of beam test scan. The left one is the "Lead-Front" configuration, and the right one is the "Lead-Rear" configuration.

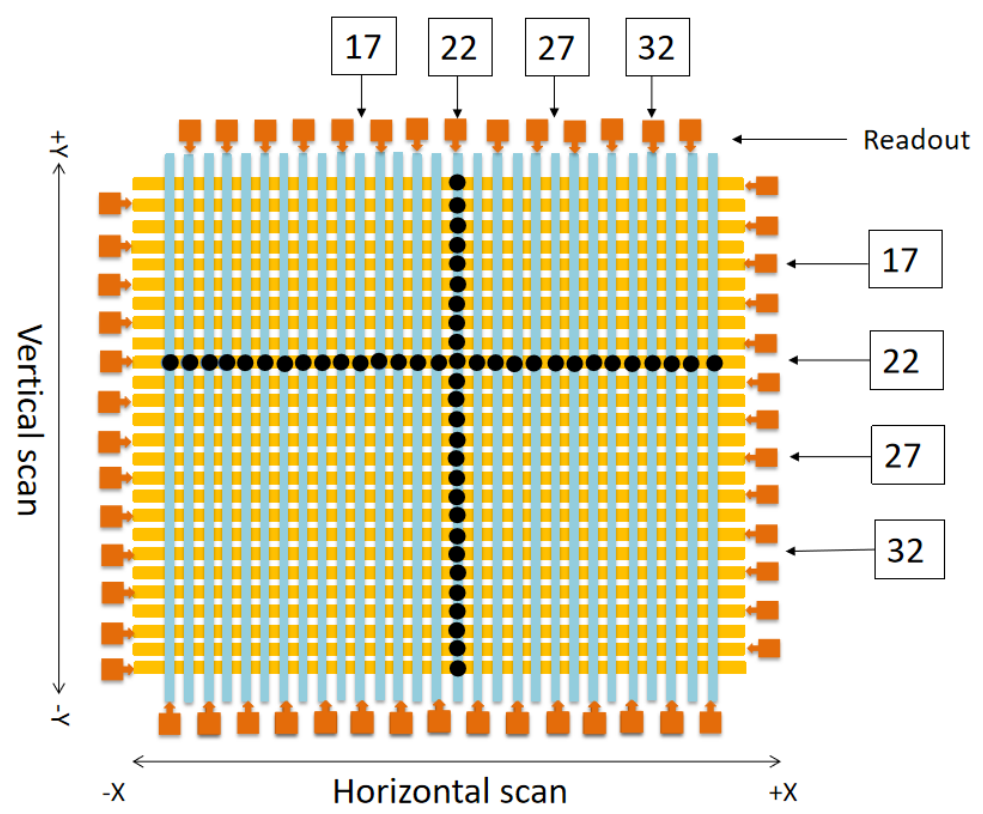

Figure 3: An illustration of the beam hit positions and part of the top two layers of the calorimeter ribbon layout. The black filled circles represent the incident electron beam spots. Layers with ribbons aligned along the $\pm Y$-direction are exposed to beams by moving the hit-position along the $\pm \mathrm{X}$-direction. Layers with ribbons aligned along the $\pm \mathrm{X}$-direction are exposed to beams by moving the hit-position along the $\pm \mathrm{Y}$-direction.

When the beam-hit ribbon position is moved along the X- or Y-direction, ribbon orthogonal to the moving direction is changed with that, but ribbons parallel to the moving direction do not change. Data were analyzed at 50 positions with $1 \mathrm{~cm}$ intervals from one end to the other, and the attenuation effect was studied using ribbons laying parallel to the moving direction in the beam-scan data. 


\section{Attenuation Effect Measurement}

Two modes are considered when the scintillation light transmitting in the fiber: the total reflection with an incident angle greater than or equal to the critical angle, and the reflection with incident angle less than the critical angle. The intensity decrease with distance to the readout $\Delta(x)$ is described by the attenuation function [6]

$$
\mathrm{A}=\mathrm{A}_{0} \times \mathrm{f}\left(\Delta(\mathrm{x}), \epsilon, \lambda_{1}, \lambda_{2}\right)=\mathrm{A}_{0} \times \epsilon \mathrm{e}^{-\Delta(\mathrm{x}) / \lambda_{1}}+\mathrm{A}_{0} \times(1-\epsilon) \mathrm{e}^{-\Delta(\mathrm{x}) / \lambda_{2}},
$$

where $\mathrm{f}\left(\Delta(\mathrm{x}), \epsilon, \lambda_{1}, \lambda_{2}\right)$ is the function related to the transmission and $\mathrm{A}_{0}$ is the intensity at the readout position. The term $\mathrm{A}_{0} \times \epsilon \mathrm{e}^{-\Delta(\mathrm{x}) / \lambda_{1}}$ represents the transmission of reflection with incident angle less than the critical angle, and $\lambda_{1}$ is the attenuation length for this mode, while the term $\mathrm{A}_{0} \times(1-\epsilon) \mathrm{e}^{-\Delta(\mathrm{x}) / \lambda_{2}}$ is the transmission behavior of total reflection, and $\lambda_{2}$ is the attenuation length for this mode. We denote $\epsilon$ as the proportion parameter for the two modes. After parameters $\left(\epsilon, \lambda_{1}\right.$, $\lambda_{2}$ ) of the attenuation lengths of ribbon 22 in all 20 layers are generated, we apply the most probable attenuation lengths, which are found to be $\lambda_{1}=3 \mathrm{~cm}$ and $\lambda_{2}=250 \mathrm{~cm}$, to all 1000 ribbons.

\section{Attenuation Effect Correction}

The energy deposit is position-dependent due to the attenuation effect. In order to correct that, we consider a primary particle at a random position $[\mathrm{x}, \mathrm{y}]$. Thus, the corrected energy deposit can be expressed as

$$
\begin{aligned}
& \mathrm{E}_{\mathrm{c}}\left(\mathrm{x}, \Delta\left(\mathrm{x}_{\mathrm{c}}\right), \Delta(\mathrm{x})\right)=\mathrm{A}(\mathrm{x}) \times \delta\left(\mathrm{x}_{\mathrm{c}}\right) \times \mathrm{f}\left(\Delta\left(\mathrm{x}_{\mathrm{c}}\right)\right) / \mathrm{f}(\Delta(\mathrm{x})) \\
& \mathrm{E}_{\mathrm{c}}\left(\mathrm{y}, \Delta\left(\mathrm{y}_{\mathrm{c}}\right), \Delta(\mathrm{y})\right)=\mathrm{A}(\mathrm{y}) \times \delta\left(\mathrm{y}_{\mathrm{c}}\right) \times \mathrm{f}\left(\Delta\left(\mathrm{y}_{\mathrm{c}}\right)\right) / \mathrm{f}(\Delta(\mathrm{y}))
\end{aligned}
$$

in even and odd layers, respectively. The signal intensities $\mathrm{A}(\mathrm{x})$ and $\mathrm{A}(\mathrm{y})$ come from the measurement of a primary particle at $[\mathrm{x}, \mathrm{y}]$, while the calibration factors, $\delta\left(\mathrm{x}_{\mathrm{c}}\right)$ and $\delta\left(\mathrm{y}_{\mathrm{c}}\right)$, come from the beam test measurement at $\left[\mathrm{x}_{\mathrm{c}}, \mathrm{y}_{\mathrm{c}}\right]$. The expressions $\mathrm{f}(\Delta(\mathrm{x})), \mathrm{f}(\Delta(\mathrm{y})), \mathrm{f}\left(\Delta\left(\mathrm{x}_{\mathrm{c}}\right)\right)$, and $\mathrm{f}\left(\Delta\left(\mathrm{y}_{\mathrm{c}}\right)\right)$ take the form of Equation (1) and parameters $\left(\epsilon, \lambda_{1}, \lambda_{2}\right)$ can be fitted. In fact, $\mathrm{f}\left(\Delta\left(\mathrm{x}_{\mathrm{c}}\right)\right)$ and $\mathrm{f}\left(\Delta\left(\mathrm{y}_{\mathrm{c}}\right)\right)$ represent the correction of the signal responses of the beam test in even and odd layers, while $f(\Delta(x))$ and $f(\Delta(y))$ represent the correction of the signal response of the primary particle.

The energy response fraction is determined by summing over all calorimeter ribbon energy deposits and dividing by the known beam test incident energy, and with that, the incident energy of a primary particle can be determined through its energy response. The energy responses for various electron energies, pion energies are shown in Section 5 and 6, while the energy response for high voltages and incident angles can be found in [7].

\section{The Energy Response for Electrons}

The ribbons' responses were measured with electron beams with increasing energies from 50 $\mathrm{GeV}$ to $175 \mathrm{GeV}$. The distribution of the energy deposits with increasing energies are shown in Fig. 4 with attenuation correction. Gaussian function fits are used to determine the energy deposits.

Fig. 5 (left) presents the energy deposit in the calorimeter as a function of the incident electron beam energy. The response is linear with increasing electron energy. With attenuation correction, 


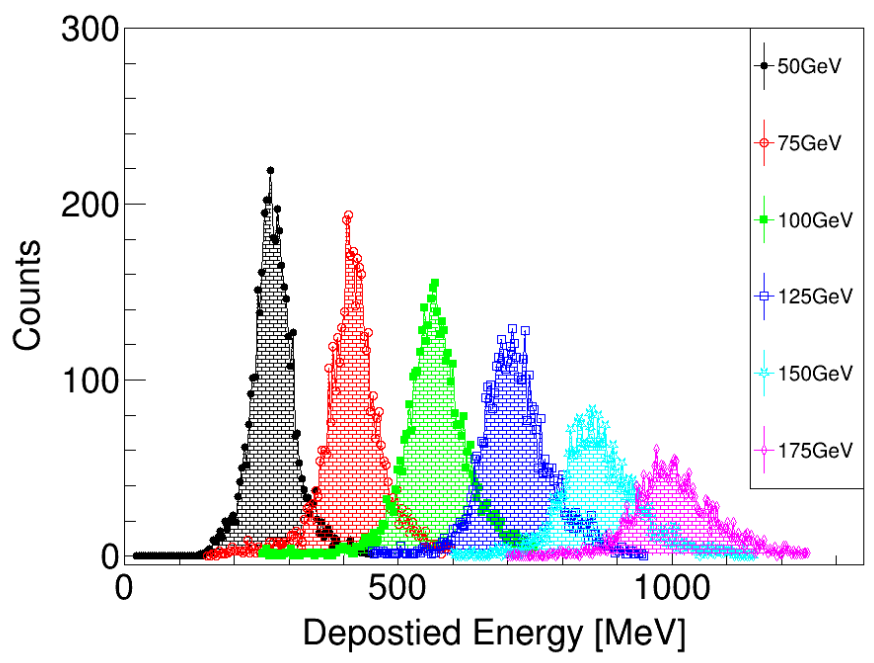

Figure 4: Energy deposited in the calorimeter by electron with beam energies of 50, 75, 100, 125, 150, and $175 \mathrm{GeV}$, which are distinguished by symbols and colors explained in the inset.
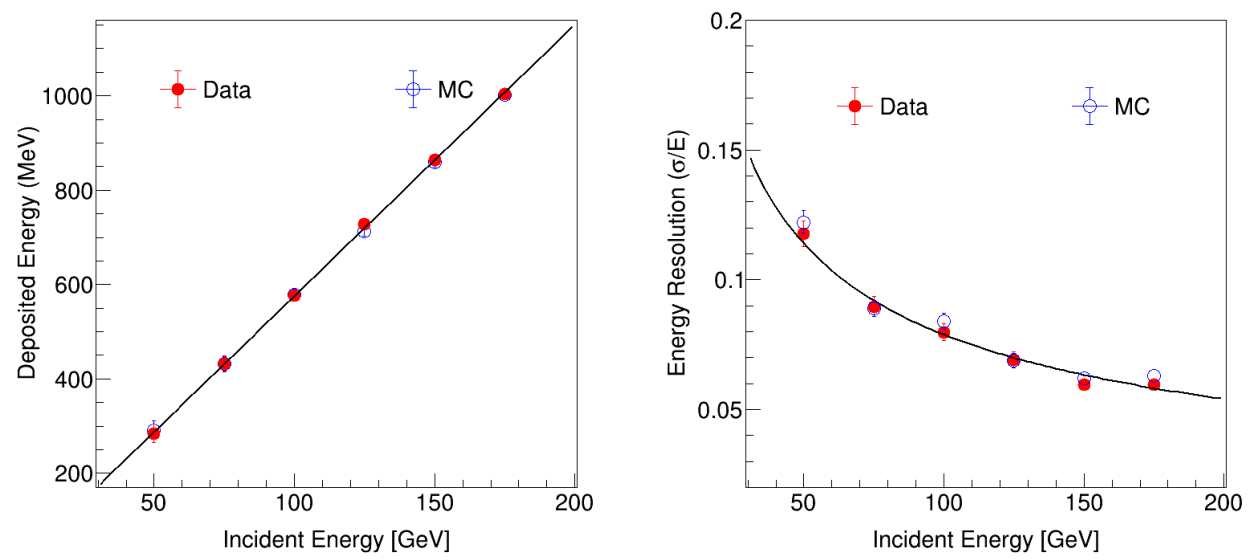

Figure 5: The mean energy deposit (left) and the energy resolutions (right) as a of the incident electron beam energies, for both the beam test data (filled red circles) and the MC simulation (open blue squares).

the slope is found to be $5.74 \mathrm{MeV} / \mathrm{GeV}$, and the total energy deposit with a $150 \mathrm{GeV}$ incident beam is $860 \mathrm{MeV}$. The result is consistent with the beam test result of the previous balloon-borne CREAM calorimeters [9].

The energy resolution with respect to the incident electron beam energy is shown in Fig. 5 (right), and it is fit to a quadratic sum function of $\sigma_{\mathrm{E}} / \mathrm{E}(\%)=85 / \sqrt{\mathrm{E}(\mathrm{GeV})} \oplus 1$.4. The energy resolution is found to be $11.4 \%$ at $50 \mathrm{GeV}$ and improves with increasing energy, reaching $6.4 \%$ at $150 \mathrm{GeV}$. The improved energy resolution at higher energy is due to the greater number of shower particles generated in the calorimeter compared with that for low energy electrons. 


\section{The Energy Response for Pions}

The ribbons' responses were measured with pion beams with energies from $250 \mathrm{GeV}$ to $350 \mathrm{GeV}$ to characterize the calorimeter's energy response to incident hadrons. A pre-selection cut requiring events with significant signals in the first three layers of the calorimeter is performed to obtain only pion events that interacted in the carbon target. The distribution of the deposited energy from pion beams is Gaussian, and as an example, the one with incident energy of $300 \mathrm{GeV}$ is shown in Fig. 6 .

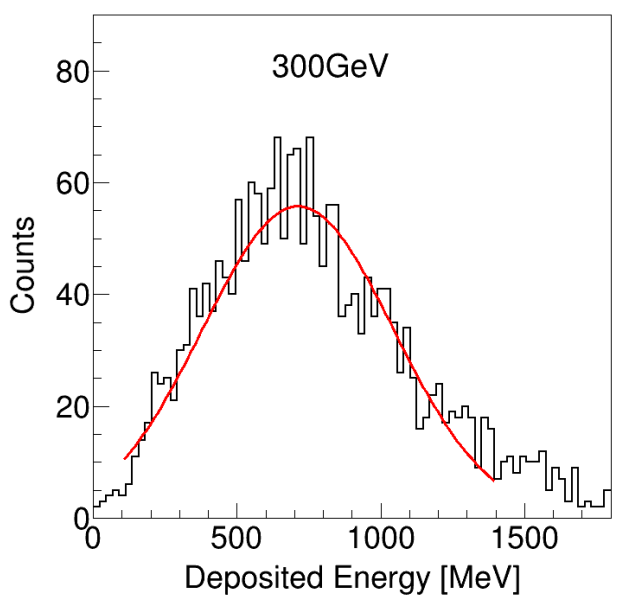

Figure 6: Energy deposits in the calorimeter for pions with the incident energy of $300 \mathrm{GeV}$. The distribution is fit to Gaussian function (red curve).
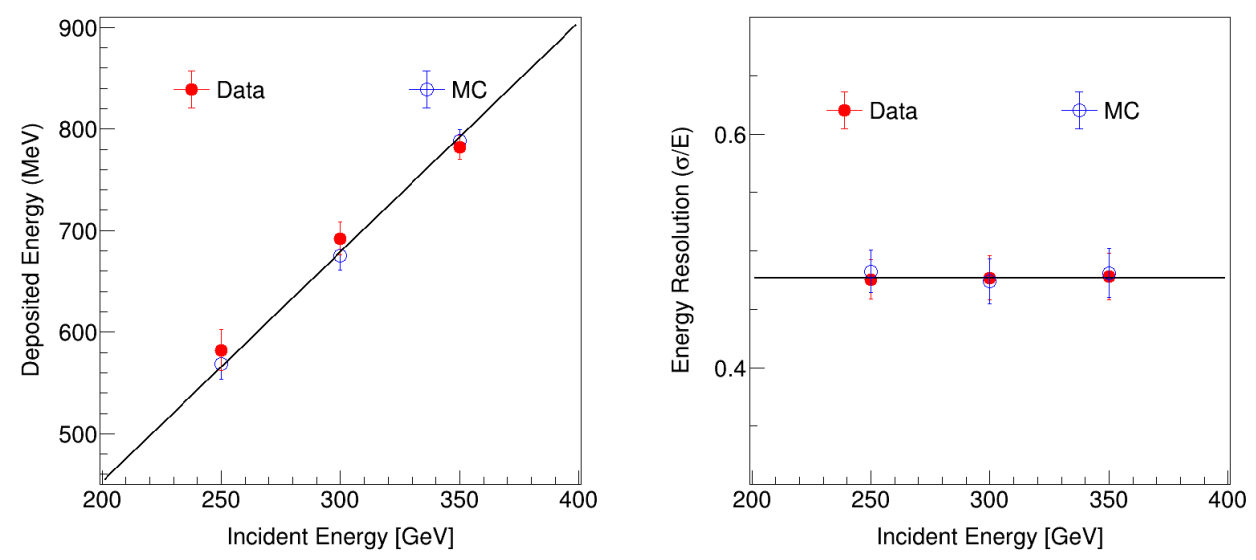

Figure 7: The mean energy deposit (left) and the energy resolution (right) as a functions of the incident pion beam energies, for both the beam test data (filled red circles) and the MC simulation (open blue squares).

Fig. 7 (left) shows the energy deposit in the calorimeter as a function of the incident pion beam energy. The response is found to be linear with attenuation correction, with a slope of $1.92 \mathrm{MeV} / \mathrm{GeV}, 1 / 3$ of the slope of the electron beam. This is consistent with theoretical expectations: when cosmic-ray particles (mainly protons) interact hadronically with the calorimeter, 
averagely $1 / 3$ of the energy of the primary nuclei are converted into neutral pions $\pi^{0}$, which then each rapidly decay into two photons [10]. The result agrees with our previous results from the beam tests of balloon-borne CREAM calorimeters [11].

The resolution as a function of the incident pion beam energy is shown in Fig. 7 (right). With attenuation correction, the resolution is found to be around $47.8 \%$, which is energy independent.

\section{Conclusion}

The tungsten scintillating-fiber calorimeter for the ISS-CREAM was calibrated with electron beams at CERN. We implemented scans of electron with a $150 \mathrm{GeV}$ electron beam to characterize its energy response. The attenuation effect due to the hit positions on fiber ribbons was corrected.

It was confirmed that the energy responses are linear for electron beams with a energy range from $50 \mathrm{GeV}$ to $175 \mathrm{GeV}$ and pion beams with an energy range from $250 \mathrm{GeV}$ to $350 \mathrm{GeV}$. These were available beams for the detector calibration at CERN, but the linearity of measurement energy range can be further validated with the heavy-ion beam test and MC simulation results [12]. The method of the attenuation correction introduced this paper are being used for flight data analysis.

\section{Acknowledgments}

This research was supported in the U.S. by NASA grant NNX17AB41G, in Korea by National Research Foundation grants (2018R1A2A1A05022685 and 2018R1A6A1A06024970) and Ministry of Science and ICT (MSIT, IITP No. 2021-0-01544 and its predecessors), and their predecessor grants. The authors also thank the support of CERN for the excellent beam test facilities and operations. H.G.Z. is grateful for the help of Dr. Zu-Hao Li.

\section{References}

[1] E. Seo et al., Cosmic Ray Energetics And Mass for the International Space Station (ISSCREAM), PoS (ICRC2019) 358 (2019) 137.

[2] S. Kang et al., On-orbit performance of the top and bottom counting detectors for the ISSCREAM experiment on the international space station, Adv. Space Res. 64 (12) (2019) 2564.

[3] G. H. Choi et al., On-Orbit Performance of the ISS-CREAM SCD, PoS(ICRC2019) 358 (2019) 048.

[4] H. Ahn et al., The cosmic ray energetics and mass (CREAM) instrument, Nucl. Instrum. Meth. A 579 (3) (2007) 1034.

[5] J. H. Han et al., A Silicon Beam Tracker, arXiv:2009.11434 [physics.ins-det].

[6] W. R. Leo, Techniques for Nuclear and Particle Physics Experiments, Springer, 1994. - 378 p.

[7] H.G. Zhang et al., Performance of the ISS-CREAM calorimeter in a calibration beam test, Astropart.Phys. 130 (2021) 102583, e-Print: 2004.10371. 
[8] M.H. Lee, et al., The CREAM calorimeter: Performance in tests and flights, Proceedings of the $12^{\text {th }}$ International Conference on Calorimetry in High Energy Physics, Chicago, AIP Conference Proceedings, 867, 2006, p. 167.

[9] J. R. Smith et al., The Cosmic Ray Energetics And Mass for the International Space Station (ISS-CREAM) Instrument, PoS (ICRC2017) 301 (2018) 199.

[10] K. Hagiwara et al., Review of Particle Properties, Phys. Rev. D 66, 010001 (2002).

[11] J. Hahn et al., Calibration of the CREAM calorimeter with beam test data, Proceedings of the 32nd International Cosmic-Ray Conference, Beijing 6 (2011) 399.

[12] H. Ahn et al., Cosmic Ray Energetics And Mass: Expected Performance, Proceedings of the 27th International Cosmic-Ray Conference, Hamburg 6 (2001) 2159. 